\title{
Cigarette smoking and depression: a question of causation ${ }^{\dagger}$
}

Marcus R. Munafò and Ricardo Araya

\begin{abstract}
Summary
Cigarette smokers frequently describe the anxiolytic and antidepressant effects of smoking, but evidence suggests that cigarette smoking may itself increase negative affect, so that the causal direction of this association remains unclear. Although increasingly sophisticated analyses of epidemiological data may help to answer this question, observational data can never unequivocally provide
\end{abstract}

evidence of causation. Here we discuss the potential utility of genetic information in determining the causal basis of the relationship of cigarette smoking and depression.

\section{Declaration of interest}

None.
Marcus R. Munafò (pictured) is Reader in Biological Psychology at the University of Bristol. His research interests are in the areas of tobacco and alcohol use, and their relationship with social cognition and emotional processing, including shared genetic influences. Ricardo Araya is Professor of Psychiatry at the University of Bristol. His research interests are in the areas of psychosocial factors associated with anxiety and depression, including social, demographic and economic inequalities and their links with the mental health of the population.

Anxiolytic and antidepressant effects of smoking are often described by cigarette smokers. Despite the strongly held popular belief that cigarette smoking can reduce negative affect, there are reports, such as that by Boden and colleagues in this issue, ${ }^{1}$ suggesting that the association is in the opposite direction, namely that smoking leads to depression. Whatever the causal direction of this association, the mechanisms which account for this high comorbidity between cigarette smoking (and tobacco use in general) and mood disorders such as major depression are not well understood. Depression may cause people to smoke (perhaps to self-medicate their symptoms), or smoking may cause increased risk of depression (via alterations in neurotransmitter pathways following chronic exposure). The relationship may even be bidirectional (acute or infrequent tobacco use may reduce negative affect, but chronic use may exacerbate it), or be caused by shared risk factors (possibly genetic) so that the relationship is not causal at all.

\section{Does smoking cause depression?}

Although evidence for a shared genetic liability for smoking and depression has been reported, ${ }^{2}$ there is growing evidence that much of the observed comorbidity may be explained by a causal effect of cigarette smoking on risk of depression. ${ }^{3}$ Boden and colleagues report further evidence in support of this explanation, using sophisticated statistical models and longitudinal data collected on adolescents and young adults. They conclude that their data are consistent with a causal model whereby cigarette smoking increases the risk of depressive symptoms. ${ }^{1}$

The use of depressive symptoms rather than diagnoses by Boden and colleagues is puzzling, given that the Composite International Diagnostic Interview (CIDI) was an instrument designed primarily to establish diagnoses. However, the authors

†ee pp. 440-446, this issue. also conducted additional testing using an ordinal variable that included three 'diagnostic' categories, reaching the same conclusions. Depressive symptoms are not the same as clinical depression, but a dimensional measure of these symptoms may offer added advantages, such as increased statistical power to test possible associations, and still represents a valid measure of negative affectivity. The choice of dependence symptoms as a measure of smoking behaviour is unusual - the number of cigarettes smoked per day might be a better indicator of exposure. Nevertheless, dependence and cigarette consumption are highly correlated, and similar effects were observed for analyses using number of cigarettes smoked per day.

\section{The paradox}

We are left with the paradox that empirical evidence suggests that smoking may cause depression, but smokers tell us that they smoke to reduce negative affect. An explanation for this paradox may come from the short half-life of nicotine and the speed with which withdrawal symptoms appear in heavy smokers who have not smoked for a few hours. Part of the withdrawal syndrome associated with acute abstinence from tobacco is increased anxiety and negative affect. Smoking will ameliorate these withdrawal symptoms. Therefore, the initial subjective experience will be one of negative affect (which will probably not be ascribed to acute withdrawal), which is subsequently reduced by smoking. It may feel like smoking reduces negative affect, but negative affect is elevated because the individual experiences the first effects of acute nicotine withdrawal. This is supported by evidence that, once the withdrawal syndrome (which typically lasts a few weeks into a cessation attempt) has ended, mood improves to a point above that experienced when the individual was smoking. ${ }^{4}$

\section{Future directions}

Ultimately, however, observational data can never unequivocally provide evidence of causation, and experimental studies are not possible for ethical reasons. What else can be done to resolve the question of direction of causation? One possible answer lies in the application of the principle of Mendelian randomisation, ${ }^{5}$ whereby genetic information can be used to test causal hypotheses regarding the effects of environmental exposures such as cigarette smoking. This requires specific polymorphisms that have been 
shown to be robustly associated with measures of exposure (e.g. number of cigarettes smoked per day). Given the random assortment of genes from parents to offspring that occurs during gamete formation and conception, genotype should not be related to potential confounders (although confounding may still occur, for example due to linkage disequilibrium with other loci, or pleiotropic effects whereby single genetic loci are associated with multiple phenotypes).

Therefore, a robust genetic influence on cigarette smoking would be akin to a randomised trial where individuals are effectively assigned to a high- or low-smoking exposure group, and could be used to test the causal relationship between smoking and depression. Although the search for the genetic antecedents of smoking behaviour has enjoyed mixed success, a small number of loci have recently emerged which appear to be convincingly associated with cigarette smoking. These include CYP2A6, which encodes the enzyme primarily responsible for the metabolism of nicotine and includes null and reduced-activity variants associated with fewer cigarettes smoked per day, ${ }^{6}$ and the CHRNA5-A3-B4 gene cluster, which encodes the alpha- 3 , alpha- 5 and beta- 4 nicotinic receptor subunits and has been associated with nicotine dependence and smoking quantity. ${ }^{7}$ Both of these might, in principle, be used to address questions of causation regarding the effects of cigarette smoking.

Some caution will be required, however; these variants should also be unlikely to be associated with other potential confounders (e.g. alcohol use) or the outcome of interest (i.e. depression) if they are to be used in this way. In other words, the genotype must only influence the outcome via effects on the exposure of interest. The CHRNA5 gene has been reported to be associated with cocaine use ${ }^{8}$ and alcohol dependence. ${ }^{9}$ Pleiotropic effects such as these are likely to be the principal limitation of the application of Mendelian randomisation to psychiatric outcomes, particularly when the genes of interest are expressed in brain.

Finally, it is worth noting that much of the recent debate around the legal status of cannabis has focused on the risks of psychiatric illness associated with prolonged use. Similarly, perhaps an underrecognised harm associated with chronic use of tobacco is an increased risk of psychiatric illness (although in this case depression rather than psychotic illness). Strong evidence that this is the case will augment existing public health messages on the risks of tobacco use, and contribute to the important and ongoing debate on the relative harms associated with the use of different psychoactive substances.

Marcus R. Munafò, PhD, Department of Experimental Psychology, University

of Bristol; Ricardo Araya, PhD, Academic Unit of Psychiatry, Department

of Community Based Medicine, University of Bristol, UK

Correspondence: Marcus R. Munafò, Department of Experimental Psychology, University of Bristol, 12a Priory Road, Bristol BS8 1TU, UK. Email: marcus.munafo@bristol.ac.uk

First received 15 Jan 2010, accepted 30 Mar 2010

\section{Funding}

This research was supported in part by a National Alliance for Research on Schizophrenia and Depression Young Investigator Award to M.M.

\section{References}

1 Boden JM, Fergusson DM, Horwood LJ. Cigarette smoking and depression: tests of causal linkage using a longitudinal birth cohort. Br J Psychiatry 2010; 196: 440-46.

2 Kendler KS, Neale MC, MacLean CJ, Heath AC, Eaves L, Kessler RC. Smoking and major depression. A causal analysis. Arch Gen Psychiatry 1993; 50: 36-43.

3 Munafò MR, Hitsman B, Rende R, Metcalfe C, Niaura R. Effects of progression to cigarette smoking on depressed mood in adolescents: evidence from the National Longitudinal Study of Adolescent Health. Addiction 2008; 103: $162-71$.

4 Munafò MR, Heron J, Araya R. Smoking patterns during pregnancy and postnatal period and depressive symptoms. Nicotine Tob Res 2008; 10: 1609-20.

5 Davey Smith G, Ebrahim S. What can mendelian randomisation tell us about modifiable behavioural and environmental exposures? BMJ 2005; 330: 1076-9.

6 Mwenifumbo JC, Tyndale RF. Genetic variability in CYP2A6 and the pharmacokinetics of nicotine. Pharmacogenomics 2007; 8: 1385-402.

7 Thorgeirsson TE, Stefansson K. Genetics of smoking behavior and its consequences: the role of nicotinic acetylcholine receptors. Biol Psychiatry 2008; 64: 919-21.

8 Grucza RA, Wang JC, Stitzel JA, Hinrichs AL, Saccone SF, Saccone NL, et al. A risk allele for nicotine dependence in CHRNA5 is a protective allele for cocaine dependence. Biol Psychiatry 2008; 64: 922-9.

9 Wang JC, Grucza R, Cruchaga C, Hinrichs AL, Bertelsen S, Budde JP, et al. Genetic variation in the CHRNA5 gene affects mRNA levels and is associated with risk for alcohol dependence. Mol Psychiatry 2009: 14: 501-10. 